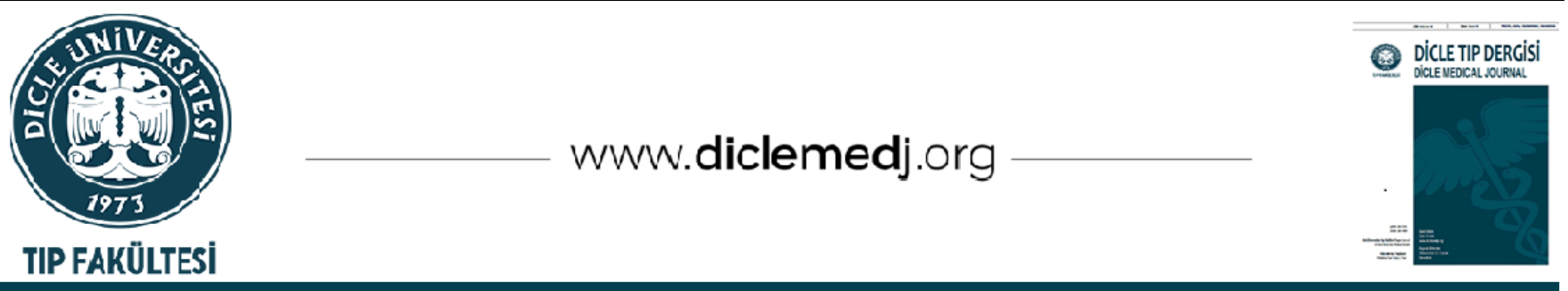

Original Article / Özgün Araştırma

\title{
In Silico Analysis of miRNA-mediated ceRNAs as Potential Molecular Biomarkers in Glioblastoma
}

\author{
Orcun Avsar ${ }^{\mathrm{D}} 1$ \\ 1 Department of Molecular Biology and Genetics, Faculty of Science and Art, Hitit University, Corum, Turkey \\ Received: 14.04.2021; Revised: 04.08.2021; Accepted: 12.08.2021
}

\begin{abstract}
Objectives: Glioblastoma multiforme (GBM) is defined as the most frequent and lethal form of the primary brain tumors in the central nervous system (CNS) in adults. Recent studies have focused on the identification of the new targets for the diagnosis and treatment of GBM and resulted in great interest for miRNAs due to their regulatory effects in cancer pathogenesis. Thus, we aimed to characterize novel molecular biomarkers for GBM by computational analysis.
\end{abstract}

Methods: 118 miRNAs that are clinically related with glioblastoma and proven by experimentally were exported through miRTarBase database. 1016 genes projected by these 118 miRNAs were determined via ComiR database. Subsequently, the genes with transcribed ultraconserved regions (T-UCRs) in their exonic regions were designated and the genes which have potential competing endogenous RNA (ceRNA) activities were extracted. Genes with remarkable expression profile differences between glioblastoma and normal brain tissues among ceRNAs that are associated with glioblastoma involving T-UCR were identified.

Results: The statistical analysis of the correlation between PBX3 and NRXN3 genes and glioblastoma was carried out by Spearman correlation test. PBX3 and NRXN3 expression was significantly higher and lower in glioblastoma than in normal brain tissues, respectively. On the other hand, the other genes did not have any remarkable differential expression pattern.

Conclusion: Based on the findings of the current study, it is determined that NRXN3 acts as a tumor suppressor gene and NRXN3 gene is downregulated in GBM. PBX3 gene functions as an oncogene and is upregulated in GBM.

Keywords: Glioblastoma, GBM, miRNA, ceRNA, T-UCR.

DOI: 10.5798/dicletip.

Correspondence / Yazıșma Adresi: Orcun Avsar, Department of Molecular Biology and Genetics, Faculty of Science and Art, Hitit University, Corum, Turkey e-mail: orcunavsar.gen@gmail.com 


\section{Glioblastomada Potansiyel Moleküler Biyobelirteçler Olarak miRNA Aracılı ceRNA'ların İn Siliko Analizi}

Öz

Amaç: Glioblastoma multiforme (GBM), yetişkinlerde santral sinir sistemi (SSS)'ndeki primer beyin tümörlerinin en sık görülen ve en öldürücü tipi olarak tanımlanmaktadır. Son yıllardaki çalışmalar, GBM'nin teşhisi ve tedavisi için yeni hedeflerin tanımlanmasına odaklanmış ve kanser patogenezindeki düzenleyici etkileri nedeniyle miRNA'lara büyük ilgi uyandırmıştır. Bu nedenle, bu çalışmada GBM için yeni moleküler biyobelirteçlerin hesaplamalı analizlerle tanımlanması amaçlanmıştır.

Yöntemler: Glioblastoma ile klinik olarak ilişkili olan ve deneysel olarak kanıtlanmış 118 miRNA, miRTarBase veri tabanından elde edildi. Elde edilen 118 miRNA tarafından hedeflenen 1016 gen ComiR veri tabanı aracılığıyla belirlendi. Akabinde, ekzonik bölgelerinde transkribe edilmiş ultra-korunmuş bölgelere (T-UCR) sahip genler belirlendi ve potansiyel olarak endojen rekabetçi RNA (ceRNA) aktivitelerine sahip olan genler ekstrakte edildi. T-UCR içeren glioblastoma ile ilişkili ceRNA'lar arasından glioblastoma ve normal beyin dokuları arasında önemli ekspresyon profili farklılıklarına sahip genler tanımlandı.

Bulgular: PBX3 ve NRXN3 genleri ile glioblastoma arasındaki korelasyonun istatistiksel analizi Spearman koralasyon testi ile gerçekleștirildi. Normal beyin dokularına göre glioblastomada PBX3 gen ekspresyonu daha yüksek iken NRXN3 gen ekspresyonu daha düşüktü. Diğer taraftan, diğer genler anlamlı farklılık gösteren ekspresyon paternine sahip değildi.

Sonuç: Mevcut çalışmanın bulgularına göre, NRXN3 geninin tümör baskılayıcı olarak işlev gördüğü ve GBM'de downregüle edildiği ve PBX3 geninin onkogen olarak görev aldığg ve GBM'de upregüle edildiği belirlendi.

Anahtar kelimeler: Glioblastoma, GBM, miRNA, ceRNA, T-UCR.

\section{INTRODUCTION}

Glioblastoma is classified as primary glioblastoma multiforme (GBM) which is seen in $80 \%$ of cases with the onset approximately at age 62, and secondary GBM which is derived from oligodendrogliomas or astrocytomas with the onset at age 45 on average ${ }^{1}$. GBM is defined as the most frequent and lethal form of the primary brain tumors in the central nervous system (CNS) in adults and classified as Grade IV by the World Health Organization ${ }^{2}$. Approximately $1 / 3$ of primary brain tumors is glioblastoma multiforme. The diagnosis and treatment of GBM is challenging and treatment options have not altered over many years even its high frequency ${ }^{3}$.

MicroRNAs (miRNAs) that are non-coding and short (18-22 nucleotides) RNA molecules are expressed in the cells of many organisms. miRNAs modulate gene and protein expression by degrading target mRNA or blocking translation. Thousands of miRNA genes have been designated in the genomes of many organisms such as plants, animals. Approximately $60 \%$ of the human genome and nearly every gene clusters are estimated to be regulated by miRNAs. MicroRNAs are key players of numerous biological functions and disruption of the function of miRNAs cause to many diseases such as cancer and neuropsychiatric diseases. Moreover, in recent years, miRNA regulation of physiology of cells, miRNA therapeutics, xenomiRs, and miRNA biomarkers have been receiving a great deal of attention by researchers ${ }^{4,5}$.

Competing endogenous RNAs (ceRNAs) are transcripts that compete for microRNA binding, modulating each other's functions posttranscriptionally. miRNAs bind to microRNA response elements (MREs) in the 3'UTRs of target mRNA. ceRNAs consist of various RNA transcript types such as protein-encoding mRNAs, circRNAs, pseudogenes, and lncRNAs. It has been proposed that many RNAs may interact with each other via MREs. The 
repressive action of miRNA is deactivated by "ceRNAs" or "miRNA sponges". The ceRNAs which have many MREs for a miRNA show multiple interactions and it is resulted with a complex regulatory network. Dysregulation of ceRNA network leads to various human diseases such as cancer. ceRNAs are key players of carcinogenesis and molecular pathways are affected by ceRNA interactions. Hence, underlying molecular mechanisms of cancer may be elucidated by the analysis of ceRNAs ${ }^{6,7}$.

Ultra-conserved regions (UCRs) are non-coding DNA sequences and conserved among mice, rats, and human beings. UCRs were discovered in mice, rats, and human genomes by bioinformatics tools in 2004. More than $90 \%$ of ultra-conserved regions are transcribed (TUCRs) in normal tissues and are modulated at the level of transcription in carcinogenesis. The expression levels of T-UCRs have tissue-specific pattern. Recent studies conducted with genome-wide expression profiling approach have demonstrated that T-UCRs show divergent profiles in various cancer types and support their roles in tumorigenesis ${ }^{8}$.

One of the most aggressive cancers, glioblastoma is challenging for treatment. Recent studies have focused on the determination of the new targets for the diagnosis and treatment of GBM and resulted in great interest for miRNAs due to their regulatory effects in normal conditions and cancer pathogenesis. According to the properties of miRNAs, it is aimed to identify novel molecular biomarkers for GBM by in silico analysis in this study.

\section{METHODS}

\section{miRNA selection}

First of all, the miRNAs that are implicated in the pathogenesis of glioblastoma were selected. In this regard, one hundred and eighteen miRNAs that are clinically related with glioblastoma and proven by experimentally were exported through miRTarBase database. Extensive information about experimentally verified miRNA-target interactions was obtained from the miRTarBase database. Scientists can apply for the database in order to confirm novel targets of miRNA ${ }^{9,10}$.

\section{miRNA-mediated ceRNAs analysis}

One thousand sixteen genes projected by these one hundred and eighteen miRNAs were determined using the ComiR database. The genes with ComiR score greater than 0.8685 were taken into account in this study. ComiR is an online web server for combinatorial miRNA target estimation and has a free access for academic users. ComiR estimates the potential of being targeted by a group of microRNAs for a mRNA in fly, mouse, worm or human genomes and each one may have zero, one or more targets on its 3'UTR. In identification the modulator potential of a mRNA from a cluster of miRNAs, ComiR uses the levels of miRNA expression which are provided by the users in a combination of relevant machine learning techniques and thermodynamic modeling in order to make more certain estimations. For each gene, the tool indicates the possibility of being functional target of a group of miRNAs according to the relative miRNA expression levels ${ }^{10-12}$. It is expected that RNA transcripts of the given genes have potential ceRNA activities for the microRNAs and this regulation may occur via miRNA-sponging mechanism ${ }^{13}$.

Matching of ceRNAs with the genes containing T-UCR

Ultra-conserved regions (UCRs) in the human genome were determined by Bejerano et al. ${ }^{14}$. The genes involving these regions classified as downstream, upstream, and exonic based on the localization within the gene ${ }^{14}$. In this study, the genes with T-UCR in their exonic regions were designated and the genes which have potential ceRNA activities were extracted in the previous analysis. 
Analysis of differential gene expression between glioblastoma and normal brain tissues

The genes with remarkable expression differences between brain tissue and glioblastoma multiforme from glioblastomaassociated ceRNAs involving T-UCR were designated via Gene Expression Profiling Interactive Analysis (GEPIA) database ${ }^{10,12}$. GEPIA that is user-friendly web tool delivers normal and cancer gene expression and interactive analysis data ${ }^{15}$.

Analysis of the correlation between PBX3 and NRXN3 genes in glioblastoma

Analysis of differential gene expression ensures to find the tumor-specific genes by comparing normal and tumor groups ${ }^{10,12}$. The statistical analysis of the relationship between PBX3 and
NRXN3 genes and glioblastoma was carried out by the use of Spearman correlation test.

\section{RESULTS}

In the current study, one hundred and eighteen miRNAs that are clinically associated with glioblastoma and proven by experimentally by the use of miRTarBase database are shown in Table I. One thousand sixteen genes that are simultaneously targeted by these 118 miRNAs were shown in supplementary I. The genes with ComiR score greater than 0.8685 were taken into account in this study. The genes which include T-UCR in exons based on the study of Bejerano et al.14 was determined and afterwards, the ones which have potential ceRNA activities were extracted and are shown in Table II.

Supplementary I: The genes targeted by these 118 glioblastoma-associated miRNAs simultaneously.

\begin{tabular}{|ll|}
\hline \multicolumn{1}{|c}{ Gene ID } & $\begin{array}{c}\text { ComiR equal } \\
\text { abundance } \\
\text { score }\end{array}$ \\
SCYL3 & 0.9157 \\
LASP1 & 0.914 \\
CFLAR & 0.9162 \\
SARM1 & 0.8693 \\
FKBP4 & 0.9142 \\
THSD7A & 0.8691 \\
KMT2E & 0.8685 \\
ZNF263 & 0.9143 \\
MAP3K9 & 0.916 \\
TTC22 & 0.9112 \\
GAS7 & 0.9159 \\
E2F2 & 0.9151 \\
CDKL5 & 0.8693 \\
ST3GAL1 & 0.8685 \\
REV3L & 0.9225 \\
IDS & 0.8685 \\
ZNF200 & 0.9121 \\
LRRC23 & 0.9112 \\
\hline
\end{tabular}

\begin{tabular}{|ll|}
\hline HFE & 0.8686 \\
SLC7A14 & 0.8689 \\
NUDCD3 & 0.8692 \\
IGF1 & 0.8691 \\
PRDM11 & 0.9222 \\
NRXN3 & 0.8686 \\
SLC45A4 & 0.9155 \\
GRAMD1B & 0.8689 \\
NDUFS1 & 0.8695 \\
KPNA6 & 0.8688 \\
$A G P A T 4$ & 0.8692 \\
$P O U 2 F 2$ & 0.9223 \\
SNX1 & 0.8694 \\
IKZF2 & 0.9157 \\
UBA6 & 0.869 \\
GAB2 & 0.9137 \\
DAPK2 & 0.869 \\
$A D A M 28$ & 0.9155 \\
HDAC9 & 0.9155 \\
SNX29 & 0.8691 \\
$R S F 1$ & 0.8691 \\
\hline
\end{tabular}

\begin{tabular}{|ll|}
\hline ADAMTS6 & 0.9156 \\
H6PD & 0.8693 \\
NEDD4L & 0.8688 \\
KIAA2022 & 0.8689 \\
HEBP2 & 0.8695 \\
MPHOSPH9 & 0.9133 \\
SIKE1 & 0.8689 \\
FOXN3 & 0.869 \\
AP5M1 & 0.9226 \\
EIF2AK2 & 0.8695 \\
KMT2C & 0.8697 \\
ATP2B4 & 0.9146 \\
RIOK2 & 0.9133 \\
BCAT1 & 0.8693 \\
MON2 & 0.9224 \\
EPN1 & 0.9162 \\
ZNF275 & 0.9153 \\
HIPK2 & 0.8695 \\
UHRF1BP1 & 0.8686 \\
GNAI3 & 0.9162 \\
WDR3 & 0.9224 \\
\hline
\end{tabular}




\begin{tabular}{|c|c|}
\hline PKN2 & 0.9145 \\
\hline$S L K$ & 0.9144 \\
\hline MTHFD2 & 0.9148 \\
\hline SLC9A7 & 0.8691 \\
\hline$C D 84$ & 0.8694 \\
\hline ATXN3 & 0.9237 \\
\hline DNTTIP2 & 0.9149 \\
\hline RRP15 & 0.869 \\
\hline ROCK1 & 0.8689 \\
\hline PSME4 & 0.9155 \\
\hline NEDD4 & 0.8691 \\
\hline GNB5 & 0.9159 \\
\hline PTPN3 & 0.9152 \\
\hline EXOC5 & 0.9159 \\
\hline RAD18 & 0.8685 \\
\hline MGAT4A & 0.8688 \\
\hline ZFYVE26 & 0.9223 \\
\hline RPS6KA6 & 0.8693 \\
\hline SMC1A & 0.9234 \\
\hline CHFR & 0.8694 \\
\hline TRHDE & 0.9161 \\
\hline P4HA2 & 0.9114 \\
\hline$I G F 2 B P 2$ & 0.9128 \\
\hline$M G L L$ & 0.9145 \\
\hline IPCEF1 & 0.869 \\
\hline$A D D 2$ & 0.8693 \\
\hline RASAL2 & 0.9162 \\
\hline ZNF37A & 0.8688 \\
\hline$F N D C 3 B$ & 0.9155 \\
\hline WDR62 & 0.8688 \\
\hline ВCAP29 & 0.9151 \\
\hline$S E C 31 B$ & 0.9151 \\
\hline RBM7 & 0.8689 \\
\hline RBMS2 & 0.8694 \\
\hline PLXNA2 & 0.869 \\
\hline PAG1 & 0.8686 \\
\hline$M B N L 3$ & 0.9162 \\
\hline PPP1R12B & 0.8696 \\
\hline
\end{tabular}

\begin{tabular}{|c|c|}
\hline DNAJC10 & 0.9162 \\
\hline$D C X$ & 0.9159 \\
\hline ACER3 & 0.8687 \\
\hline РIKЗСЗ & 0.8685 \\
\hline$N 4 B P 2$ & 0.8691 \\
\hline RUNX1T1 & 0.8687 \\
\hline RIF1 & 0.8694 \\
\hline$R A B 21$ & 0.8696 \\
\hline $\mathrm{CDH7}$ & 0.9161 \\
\hline$M E F 2 C$ & 0.9134 \\
\hline$B Z W 1$ & 0.9119 \\
\hline$P G R$ & 0.8695 \\
\hline FAM135A & 0.9158 \\
\hline ERC1 & 0.8687 \\
\hline XP01 & 0.869 \\
\hline LYRM2 & 0.9157 \\
\hline ZNF264 & 0.9162 \\
\hline SSH1 & 0.8696 \\
\hline MAPЗK4 & 0.9134 \\
\hline PTPN4 & 0.8687 \\
\hline C20orf194 & 0.9145 \\
\hline MAVS & 0.8696 \\
\hline NOS1 & 0.8689 \\
\hline ZBTB25 & 0.9161 \\
\hline ARHGAP4 & 0.9143 \\
\hline GPATCH2L & 0.9162 \\
\hline PCBP4 & 0.9199 \\
\hline ZNF268 & 0.9224 \\
\hline$P D P R$ & 0.8686 \\
\hline TNRC6A & 0.8695 \\
\hline DTX2 & 0.9096 \\
\hline RGS17 & 0.8685 \\
\hline SNAP23 & 0.9116 \\
\hline$A G 01$ & 0.9162 \\
\hline GPATCH 2 & 0.9156 \\
\hline ECHDC1 & 0.9118 \\
\hline CBX5 & 0.9226 \\
\hline FKBP5 & 0.8692 \\
\hline
\end{tabular}

\begin{tabular}{|c|c|}
\hline$C D C 5 L$ & 0.9142 \\
\hline$C D C 34$ & 0.9106 \\
\hline MTAP & 0.9157 \\
\hline CECR2 & 0.915 \\
\hline$D D T L$ & 0.9106 \\
\hline MAPK1 & 0.8695 \\
\hline$A D R B K 2$ & 0.8692 \\
\hline TFIP11 & 0.91 \\
\hline RBFOX2 & 0.8688 \\
\hline MTMR3 & 0.8687 \\
\hline MIEF1 & 0.9142 \\
\hline KIAA0930 & 0.9151 \\
\hline DDHD1 & 0.9162 \\
\hline VTI1B & 0.8685 \\
\hline SPTLC2 & 0.8688 \\
\hline GALNT16 & 0.9152 \\
\hline DICER1 & 0.9155 \\
\hline ZССH14 & 0.9162 \\
\hline RPS6KA5 & 0.9237 \\
\hline KIAA0391 & 0.9219 \\
\hline SLC52A3 & 0.9124 \\
\hline ST8SIA5 & 0.8695 \\
\hline CEP192 & 0.8693 \\
\hline$R N M T$ & 0.9154 \\
\hline$L I P G$ & 0.8695 \\
\hline$A N K R D 12$ & 0.915 \\
\hline MIB1 & 0.915 \\
\hline PGRMC1 & 0.9103 \\
\hline$A L G 13$ & 0.9151 \\
\hline PORCN & 0.9126 \\
\hline$K L F 8$ & 0.9149 \\
\hline$F G F 14$ & 0.9161 \\
\hline$F N D C 3 A$ & 0.915 \\
\hline STK24 & 0.9235 \\
\hline KATNAL1 & 0.9152 \\
\hline INTS6 & 0.8696 \\
\hline NFAT5 & 0.8696 \\
\hline LONP2 & 0.8687 \\
\hline
\end{tabular}




\begin{tabular}{|c|c|}
\hline$C C D C 113$ & 0.9147 \\
\hline SLC7A6 & 0.9157 \\
\hline ESRP2 & 0.9134 \\
\hline$M L Y C D$ & 0.8694 \\
\hline GSPT1 & 0.8689 \\
\hline$G G A 2$ & 0.9152 \\
\hline XYLT1 & 0.8694 \\
\hline HOMER2 & 0.916 \\
\hline EHD4 & 0.8687 \\
\hline ATP8B4 & 0.8687 \\
\hline DTWD1 & 0.8696 \\
\hline SLC30A4 & 0.8686 \\
\hline$M Y E F 2$ & 0.8689 \\
\hline FZD3 & 0.9226 \\
\hline$U B E 2 W$ & 0.9161 \\
\hline$T U B B 4 A$ & 0.9126 \\
\hline AKAP8 & 0.9139 \\
\hline$A V L 9$ & 0.8687 \\
\hline CDK6 & 0.8694 \\
\hline ITGB8 & 0.869 \\
\hline TTC26 & 0.914 \\
\hline TFEC & 0.8685 \\
\hline HOXA1 & 0.9116 \\
\hline PLEKHA8 & 0.9158 \\
\hline$A P 1 S 1$ & 0.9112 \\
\hline C1GALT1 & 0.8688 \\
\hline TMEM106B & 0.8696 \\
\hline FKTN & 0.9224 \\
\hline TGFBR1 & 0.915 \\
\hline$A K N A$ & 0.9146 \\
\hline KCNT1 & 0.9222 \\
\hline$R G P 1$ & 0.8686 \\
\hline$A B C A 2$ & 0.8685 \\
\hline CCNJ & 0.9133 \\
\hline PLEKHA1 & 0.8696 \\
\hline$B M P R 1 A$ & 0.916 \\
\hline СРЕВ3 & 0.9158 \\
\hline$F B X L 20$ & 0.8695 \\
\hline
\end{tabular}

\begin{tabular}{|c|c|}
\hline INTS2 & 0.9114 \\
\hline LUC7L3 & 0.8688 \\
\hline GABRA4 & 0.8695 \\
\hline CLNK & 0.9142 \\
\hline CTSC & 0.869 \\
\hline DTX4 & 0.9148 \\
\hline CCND1 & 0.9122 \\
\hline$C B L$ & 0.9161 \\
\hline CARS & 0.913 \\
\hline SOX6 & 0.8691 \\
\hline CAPRIN2 & 0.9159 \\
\hline DUSP16 & 0.9143 \\
\hline C12orf49 & 0.8694 \\
\hline TBC1D30 & 0.9156 \\
\hline CNOT2 & 0.9129 \\
\hline KRR1 & 0.869 \\
\hline ST8SIA1 & 0.869 \\
\hline$F R K$ & 0.8696 \\
\hline SOD2 & 0.8692 \\
\hline$R N F 8$ & 0.8687 \\
\hline ZNF451 & 0.8694 \\
\hline ASCC 3 & 0.9139 \\
\hline KIAA1244 & 0.8694 \\
\hline SLC16A10 & 0.9225 \\
\hline IMPG1 & 0.869 \\
\hline$G H R$ & 0.9153 \\
\hline COL4A3BP & 0.9149 \\
\hline PRLR & 0.9225 \\
\hline SKP1 & 0.9161 \\
\hline CPEB4 & 0.9146 \\
\hline KPNA1 & 0.9156 \\
\hline UBE3A & 0.9221 \\
\hline XRN1 & 0.9154 \\
\hline$B B X$ & 0.9159 \\
\hline KIAA1257 & 0.8685 \\
\hline HEMK1 & 0.9241 \\
\hline$A C V R 2 B$ & 0.9161 \\
\hline$A B C C 5$ & 0.9144 \\
\hline
\end{tabular}

\begin{tabular}{|c|c|}
\hline KLHL24 & 0.9152 \\
\hline INO80D & 0.8696 \\
\hline$T T L$ & 0.9161 \\
\hline TFCP2L1 & 0.8693 \\
\hline DNAJC27 & 0.8685 \\
\hline$A P C 2$ & 0.9142 \\
\hline ТТС31 & 0.9139 \\
\hline$P A P O L G$ & 0.8685 \\
\hline ELMOD3 & 0.9131 \\
\hline$G G C X$ & 0.8692 \\
\hline ZNF142 & 0.8691 \\
\hline$H D L B P$ & 0.9124 \\
\hline PLCL1 & 0.8692 \\
\hline$K Y N U$ & 0.8696 \\
\hline$A A K 1$ & 0.9162 \\
\hline ARID3A & 0.9209 \\
\hline PLEKHAЗ & 0.9237 \\
\hline$T N R$ & 0.8694 \\
\hline$G P X 7$ & 0.9113 \\
\hline KCNC4 & 0.8697 \\
\hline$M E F 2 D$ & 0.9156 \\
\hline C1orf21 & 0.9162 \\
\hline TROVE2 & 0.9155 \\
\hline MTR & 0.8691 \\
\hline RIMS3 & 0.869 \\
\hline AKT3 & 0.8688 \\
\hline CTBS & 0.8688 \\
\hline TMED5 & 0.8686 \\
\hline$D R 1$ & 0.8695 \\
\hline РТВР2 & 0.8696 \\
\hline DIEXF & 0.8693 \\
\hline SLC5A9 & 0.9113 \\
\hline SGIP1 & 0.8688 \\
\hline$A D G B$ & 0.916 \\
\hline MED28 & 0.9162 \\
\hline SLC16A7 & 0.8696 \\
\hline DCLRE1B & 0.9138 \\
\hline CCND2 & 0.9222 \\
\hline
\end{tabular}




\begin{tabular}{|c|c|}
\hline CYP20A1 & 0.8695 \\
\hline TRPM6 & 0.9135 \\
\hline TRIM67 & 0.9157 \\
\hline$F B X W 2$ & 0.9161 \\
\hline RBM18 & 0.8686 \\
\hline ONECUT2 & 0.9241 \\
\hline YLPM1 & 0.9152 \\
\hline NEK9 & 0.9154 \\
\hline DNAL1 & 0.8693 \\
\hline NRDE2 & 0.8695 \\
\hline ZNF410 & 0.9126 \\
\hline YIPF4 & 0.8695 \\
\hline FAM178A & 0.9146 \\
\hline HELLS & 0.9155 \\
\hline МОВЗВ & 0.869 \\
\hline$B 4 G A L T 4$ & 0.8686 \\
\hline ACVR2A & 0.9152 \\
\hline$O D F 2 L$ & 0.8685 \\
\hline ZNF644 & 0.9106 \\
\hline SEPT7 & 0.8689 \\
\hline CHST3 & 0.915 \\
\hline SLC25A16 & 0.8686 \\
\hline SPRYD7 & 0.9117 \\
\hline$N L N$ & 0.9219 \\
\hline ATPAF1 & 0.9145 \\
\hline ACVR1C & 0.8687 \\
\hline LPGAT1 & 0.9224 \\
\hline PARD 6B & 0.9151 \\
\hline$R A B 22 A$ & 0.8691 \\
\hline BCAS4 & 0.8689 \\
\hline STAMBP & 0.8687 \\
\hline HIF3A & 0.9155 \\
\hline NQO2 & 0.916 \\
\hline ATXN1 & 0.8694 \\
\hline SH3TC1 & 0.9219 \\
\hline ATP5S & 0.9225 \\
\hline GGA3 & 0.9154 \\
\hline GTF3C4 & 0.8688 \\
\hline
\end{tabular}

\begin{tabular}{|c|c|}
\hline POLR1B & 0.9153 \\
\hline THOC2 & 0.9141 \\
\hline$M E D 1$ & 0.8686 \\
\hline GPCPD1 & 0.915 \\
\hline TMX4 & 0.9152 \\
\hline AP5S1 & 0.9155 \\
\hline$M K K S$ & 0.9155 \\
\hline$R A L Y$ & 0.9223 \\
\hline CEP250 & 0.916 \\
\hline$A M O T$ & 0.9155 \\
\hline$A G O 3$ & 0.9226 \\
\hline THRA & 0.9143 \\
\hline PCNXL4 & 0.9162 \\
\hline MASP1 & 0.915 \\
\hline$H E L B$ & 0.9161 \\
\hline$R A P 1 B$ & 0.8696 \\
\hline$R A B 3 I P$ & 0.8691 \\
\hline PTPRB & 0.869 \\
\hline DYRK2 & 0.9158 \\
\hline ZNF835 & 0.9126 \\
\hline HIP1 & 0.8688 \\
\hline FOXP2 & 0.9224 \\
\hline$M K L N 1$ & 0.9161 \\
\hline TMOD2 & 0.8693 \\
\hline ICE2 & 0.9158 \\
\hline ARPP19 & 0.869 \\
\hline CALML4 & 0.9137 \\
\hline KCNC1 & 0.8689 \\
\hline PRRG3 & 0.8685 \\
\hline АТР8BЗ & 0.9158 \\
\hline$D D A 1$ & 0.9155 \\
\hline TULP4 & 0.8689 \\
\hline$P X D N$ & 0.9132 \\
\hline PGPEP1 & 0.9156 \\
\hline ZNF557 & 0.8687 \\
\hline ZNF341 & 0.9215 \\
\hline NFATC1 & 0.9121 \\
\hline RAB11FIP4 & 0.9154 \\
\hline
\end{tabular}

\begin{tabular}{|c|c|}
\hline LRRC41 & 0.9107 \\
\hline ENOSF1 & 0.9147 \\
\hline GRSF1 & 0.8688 \\
\hline PCBD2 & 0.869 \\
\hline SCO1 & 0.8693 \\
\hline STARD13 & 0.9137 \\
\hline$L A R G E$ & 0.8687 \\
\hline MYO18B & 0.8687 \\
\hline FAM83F & 0.8697 \\
\hline$M B D 2$ & 0.9218 \\
\hline WNT2B & 0.916 \\
\hline$M Y C N$ & 0.9119 \\
\hline CRB1 & 0.9223 \\
\hline KLRD1 & 0.9237 \\
\hline AGO4 & 0.9153 \\
\hline BTF3L4 & 0.8685 \\
\hline$D A G L A$ & 0.9156 \\
\hline$F A D S 2$ & 0.9131 \\
\hline CLOCK & 0.9159 \\
\hline DZIP1 & 0.8685 \\
\hline MTO1 & 0.9161 \\
\hline ZСЗН10 & 0.8693 \\
\hline$C D 164$ & 0.914 \\
\hline REPS1 & 0.8687 \\
\hline USP15 & 0.8696 \\
\hline$C P M$ & 0.8689 \\
\hline KIAA0513 & 0.8693 \\
\hline SLC9A5 & 0.9149 \\
\hline$R C 3 H 1$ & 0.8692 \\
\hline TTLL4 & 0.9121 \\
\hline$A L D H 1 L 2$ & 0.9158 \\
\hline USP44 & 0.9192 \\
\hline$S L C 41 A 2$ & 0.9146 \\
\hline$A L P K 3$ & 0.8687 \\
\hline LIMD2 & 0.9224 \\
\hline KAT7 & 0.8695 \\
\hline SKIL & 0.922 \\
\hline UGGT1 & 0.9157 \\
\hline
\end{tabular}




\begin{tabular}{|c|c|}
\hline ARHGEF39 & 0.9131 \\
\hline YIPF3 & 0.9113 \\
\hline SLC22A23 & 0.8688 \\
\hline TAF8 & 0.9159 \\
\hline CPEB2 & 0.9205 \\
\hline$R A B 30$ & 0.8694 \\
\hline SLCO5A1 & 0.9155 \\
\hline TMPRSS4 & 0.8686 \\
\hline UNC13C & 0.9159 \\
\hline CTDSPL2 & 0.9151 \\
\hline THBS1 & 0.8687 \\
\hline ITGA11 & 0.9223 \\
\hline$A D A M 10$ & 0.8695 \\
\hline TTLL7 & 0.869 \\
\hline IFI44L & 0.9152 \\
\hline ADAMTS14 & 0.9123 \\
\hline SSFA2 & 0.9157 \\
\hline$A B I 2$ & 0.8697 \\
\hline PARP9 & 0.9151 \\
\hline CNOT6L & 0.8689 \\
\hline KIAA1644 & 0.922 \\
\hline NDUFA9 & 0.8686 \\
\hline TARBP2 & 0.9126 \\
\hline ACVRL1 & 0.913 \\
\hline$A N K R D 52$ & 0.9234 \\
\hline ZNF740 & 0.9158 \\
\hline WDFY2 & 0.8691 \\
\hline NOVA1 & 0.9159 \\
\hline SYT16 & 0.8696 \\
\hline SLC38A6 & 0.9145 \\
\hline NAA30 & 0.9234 \\
\hline$R A B 15$ & 0.9215 \\
\hline TSPAN3 & 0.9159 \\
\hline$I G F 1 R$ & 0.9224 \\
\hline ABHD2 & 0.8693 \\
\hline NTRK3 & 0.9226 \\
\hline DET1 & 0.9138 \\
\hline ZNF710 & 0.9147 \\
\hline
\end{tabular}

\begin{tabular}{|c|c|}
\hline FTO & 0.9161 \\
\hline NKD1 & 0.8691 \\
\hline GFOD2 & 0.9156 \\
\hline РСТP & 0.9129 \\
\hline$G N A L$ & 0.8685 \\
\hline C18orf21 & 0.9084 \\
\hline GALNT1 & 0.9129 \\
\hline GAREM & 0.8685 \\
\hline TP53 & 0.913 \\
\hline$T B C D$ & 0.9108 \\
\hline TRIM65 & 0.9114 \\
\hline RNF165 & 0.9161 \\
\hline WTIP & 0.8695 \\
\hline POU2F1 & 0.9237 \\
\hline$A B L 2$ & 0.916 \\
\hline RGS16 & 0.9116 \\
\hline$L H X 9$ & 0.9155 \\
\hline SNX27 & 0.8693 \\
\hline$G A B P B 2$ & 0.916 \\
\hline SYT14 & 0.8691 \\
\hline$A C P 1$ & 0.9116 \\
\hline PLEKHA6 & 0.9156 \\
\hline PTPN7 & 0.9114 \\
\hline SYT2 & 0.8691 \\
\hline TEX261 & 0.9145 \\
\hline ZСЗН8 & 0.9158 \\
\hline KIAA1715 & 0.8692 \\
\hline GULP1 & 0.869 \\
\hline SPAG16 & 0.922 \\
\hline LIMD1 & 0.9161 \\
\hline ZNF660 & 0.9158 \\
\hline MUC4 & 0.9146 \\
\hline TBCK & 0.869 \\
\hline SPATA5 & 0.8689 \\
\hline METTL14 & 0.9157 \\
\hline USP53 & 0.9148 \\
\hline UGT3A1 & 0.9154 \\
\hline SSBP2 & 0.869 \\
\hline
\end{tabular}

\begin{tabular}{|c|c|}
\hline PPIP5K2 & 0.8696 \\
\hline$B D P 1$ & 0.9145 \\
\hline TNFAIPB & 0.9159 \\
\hline ATG12 & 0.9151 \\
\hline ARHGAP26 & 0.8693 \\
\hline PCYOX1L & 0.9138 \\
\hline G3BP1 & 0.869 \\
\hline GFOD1 & 0.8691 \\
\hline IRAK1BP1 & 0.8687 \\
\hline$M M S 22 L$ & 0.9155 \\
\hline FAXC & 0.8694 \\
\hline CLVS2 & 0.8695 \\
\hline RNF217 & 0.9225 \\
\hline SHPRH & 0.8695 \\
\hline PURB & 0.8691 \\
\hline CASK & 0.8691 \\
\hline$K D M 6 A$ & 0.9143 \\
\hline DIAPH2 & 0.916 \\
\hline FAM135B & 0.9154 \\
\hline$V L D L R$ & 0.916 \\
\hline$U G C G$ & 0.9136 \\
\hline SNX30 & 0.9221 \\
\hline NR6A1 & 0.9237 \\
\hline$A 1 C F$ & 0.9225 \\
\hline EIF4EBP2 & 0.8688 \\
\hline CNNM2 & 0.8696 \\
\hline INTS4 & 0.9135 \\
\hline SOGA1 & 0.8695 \\
\hline PCDH15 & 0.8689 \\
\hline СDН8 & 0.9154 \\
\hline LPHN3 & 0.916 \\
\hline$P D C D 4$ & 0.913 \\
\hline CD226 & 0.8696 \\
\hline FREM2 & 0.8691 \\
\hline$D C P 1 B$ & 0.9121 \\
\hline THRB & 0.8689 \\
\hline GXYLT1 & 0.9158 \\
\hline AKAP6 & 0.9161 \\
\hline
\end{tabular}




\begin{tabular}{|c|c|}
\hline THRSP & 0.9104 \\
\hline ADAMTS12 & 0.9151 \\
\hline C4orf33 & 0.8689 \\
\hline$W W C 2$ & 0.8686 \\
\hline GABRA2 & 0.8688 \\
\hline GFRA1 & 0.8695 \\
\hline CACUL1 & 0.8694 \\
\hline$R A B G A P 1 L$ & 0.8687 \\
\hline PTPN14 & 0.8694 \\
\hline EPG5 & 0.8689 \\
\hline ATP5A1 & 0.8686 \\
\hline GUCY1A2 & 0.9226 \\
\hline ZNF773 & 0.8692 \\
\hline FARP1 & 0.9157 \\
\hline ZNF117 & 0.8685 \\
\hline SREK1IP1 & 0.9223 \\
\hline SMARCA5 & 0.8685 \\
\hline$R A N B P 2$ & 0.9117 \\
\hline$A S A P 1$ & 0.9222 \\
\hline PTPRD & 0.9149 \\
\hline CNKSR3 & 0.9237 \\
\hline SREK1 & 0.8686 \\
\hline HS2ST1 & 0.869 \\
\hline MSI2 & 0.8689 \\
\hline CHST9 & 0.9162 \\
\hline OTULIN & 0.8685 \\
\hline LRRK1 & 0.9162 \\
\hline ENAH & 0.9161 \\
\hline GPR26 & 0.8691 \\
\hline ADAMTS5 & 0.8688 \\
\hline PIEZO2 & 0.8687 \\
\hline$A P O O L$ & 0.869 \\
\hline ATP6V1C1 & 0.9151 \\
\hline PDZD9 & 0.9115 \\
\hline PPARGC1B & 0.9224 \\
\hline LSM11 & 0.8689 \\
\hline$A F F 2$ & 0.8693 \\
\hline PSD3 & 0.8694 \\
\hline
\end{tabular}

\begin{tabular}{|c|c|}
\hline MMP16 & 0.8693 \\
\hline BACH1 & 0.9127 \\
\hline ANKRD9 & 0.8686 \\
\hline UQCRB & 0.8688 \\
\hline AIFM1 & 0.9129 \\
\hline FBXO32 & 0.8689 \\
\hline B3GNT7 & 0.9134 \\
\hline$A T P 2 B 2$ & 0.8685 \\
\hline STEAP2 & 0.8689 \\
\hline HYDIN & 0.9151 \\
\hline$M Y 01 E$ & 0.8687 \\
\hline KCNJ6 & 0.8696 \\
\hline TSPAN18 & 0.9152 \\
\hline$D G K I$ & 0.8696 \\
\hline UBN2 & 0.8695 \\
\hline$B R A F$ & 0.8693 \\
\hline AP3S2 & 0.8688 \\
\hline WIPI2 & 0.9142 \\
\hline PAFAH2 & 0.914 \\
\hline$X K R 8$ & 0.9115 \\
\hline EYA3 & 0.8687 \\
\hline CLSTN2 & 0.8696 \\
\hline PPP1R15B & 0.9142 \\
\hline AGPAT6 & 0.9151 \\
\hline$E L K 4$ & 0.9161 \\
\hline TNNI1 & 0.869 \\
\hline$I G F 2 B P 1$ & 0.9235 \\
\hline$S C U B E 1$ & 0.8692 \\
\hline STARD9 & 0.9204 \\
\hline$A C E$ & 0.9124 \\
\hline$B S D C 1$ & 0.9125 \\
\hline$Z B T B 8 A$ & 0.869 \\
\hline ZNF362 & 0.9125 \\
\hline TRAPPC10 & 0.8685 \\
\hline ICOSLG & 0.9152 \\
\hline TAOK1 & 0.8691 \\
\hline MFSD12 & 0.9099 \\
\hline PLXDC1 & 0.8686 \\
\hline
\end{tabular}

\begin{tabular}{|c|c|}
\hline IKZF3 & 0.9161 \\
\hline ACOX1 & 0.8688 \\
\hline TMEM143 & 0.9099 \\
\hline FMNL3 & 0.9162 \\
\hline TREML1 & 0.9102 \\
\hline$C C N F$ & 0.9226 \\
\hline PRKAA2 & 0.8693 \\
\hline CTRC & 0.9147 \\
\hline RBBP4 & 0.916 \\
\hline UBXN10 & 0.8686 \\
\hline NFIA & 0.8693 \\
\hline ZNF326 & 0.8693 \\
\hline SLC30A7 & 0.8689 \\
\hline VANGL2 & 0.914 \\
\hline ACP6 & 0.9234 \\
\hline WDR26 & 0.8686 \\
\hline$R E L$ & 0.9162 \\
\hline DISC1 & 0.8688 \\
\hline FAM84A & 0.869 \\
\hline DUSP19 & 0.8685 \\
\hline SMARCAD1 & 0.9141 \\
\hline$E O G T$ & 0.9132 \\
\hline EIF4E3 & 0.8694 \\
\hline LRRC58 & 0.8692 \\
\hline$C C D C 141$ & 0.8686 \\
\hline$I C A 1 L$ & 0.8692 \\
\hline$R Y B P$ & 0.9155 \\
\hline$R P P 14$ & 0.8692 \\
\hline RBM47 & 0.9146 \\
\hline$A P B B 2$ & 0.9158 \\
\hline TTC14 & 0.9158 \\
\hline SENP2 & 0.9151 \\
\hline IFT122 & 0.9153 \\
\hline SFMBT1 & 0.9111 \\
\hline$C D C 25 A$ & 0.9184 \\
\hline INTU & 0.8696 \\
\hline RNF123 & 0.9139 \\
\hline MFSD8 & 0.9144 \\
\hline
\end{tabular}




\begin{tabular}{|c|c|}
\hline$W D R 41$ & 0.9142 \\
\hline GPX8 & 0.9147 \\
\hline RICTOR & 0.8692 \\
\hline$D C B L D 1$ & 0.9118 \\
\hline KIF6 & 0.8685 \\
\hline USP49 & 0.8692 \\
\hline$D L C 1$ & 0.913 \\
\hline$A D C Y 1$ & 0.8694 \\
\hline TP53INP1 & 0.8685 \\
\hline KIAA1958 & 0.9225 \\
\hline STRBP & 0.9147 \\
\hline$H D X$ & 0.9151 \\
\hline BRWD3 & 0.8685 \\
\hline SLITRK5 & 0.9226 \\
\hline CFL2 & 0.8691 \\
\hline SUGT1 & 0.9162 \\
\hline PGM2L1 & 0.9154 \\
\hline SLC16A9 & 0.9141 \\
\hline AMER2 & 0.8695 \\
\hline PDZD8 & 0.8692 \\
\hline FAM204A & 0.8696 \\
\hline CLEC1B & 0.9142 \\
\hline FUNDC2 & 0.8689 \\
\hline$A G B L 2$ & 0.919 \\
\hline CPSF2 & 0.9162 \\
\hline$A R L 5 B$ & 0.8689 \\
\hline ADAMTS15 & 0.9135 \\
\hline HIF1AN & 0.9226 \\
\hline SPINT1 & 0.9096 \\
\hline ARIH1 & 0.9237 \\
\hline SYNPO2L & 0.9135 \\
\hline TRIM44 & 0.8696 \\
\hline TPP1 & 0.9134 \\
\hline TRIM66 & 0.8692 \\
\hline$P R T G$ & 0.9225 \\
\hline PKD1L2 & 0.9138 \\
\hline$N A$ & 0.9224 \\
\hline TMED3 & 0.8696 \\
\hline
\end{tabular}

\begin{tabular}{|c|c|}
\hline GALR1 & 0.9161 \\
\hline TVP23A & 0.9143 \\
\hline SLFN5 & 0.8689 \\
\hline GREM1 & 0.8696 \\
\hline SGSM1 & 0.9145 \\
\hline$P B X 3$ & 0.9124 \\
\hline FBXO22 & 0.9162 \\
\hline$I R G Q$ & 0.916 \\
\hline ZNF226 & 0.9155 \\
\hline$A N K R D 11$ & 0.8691 \\
\hline ZNF641 & 0.9223 \\
\hline TTYH1 & 0.9213 \\
\hline$M A P K 1 I P 1 L$ & 0.9159 \\
\hline POLR3D & 0.9216 \\
\hline FAM84B & 0.9151 \\
\hline TET2 & 0.9152 \\
\hline$A N K R D 49$ & 0.914 \\
\hline IRS1 & 0.8688 \\
\hline MECP2 & 0.8692 \\
\hline$R A B 3 B$ & 0.8696 \\
\hline SH3TC2 & 0.8694 \\
\hline SHE & 0.8688 \\
\hline PTAFR & 0.9212 \\
\hline HIC2 & 0.923 \\
\hline TOR1AIP2 & 0.8691 \\
\hline МАРЗК2 & 0.869 \\
\hline TMEM154 & 0.8695 \\
\hline GPR37L1 & 0.8689 \\
\hline TMEM192 & 0.9225 \\
\hline NIPA1 & 0.9152 \\
\hline RNF150 & 0.9161 \\
\hline USP38 & 0.9233 \\
\hline CRTAP & 0.8687 \\
\hline KRT78 & 0.9121 \\
\hline LONRF2 & 0.8692 \\
\hline SERPINB9 & 0.9147 \\
\hline NUDCD2 & 0.9224 \\
\hline$S G C D$ & 0.8692 \\
\hline
\end{tabular}

\begin{tabular}{|c|c|}
\hline ATF7 & 0.9155 \\
\hline TMEM126B & 0.911 \\
\hline NETO2 & 0.8688 \\
\hline CLCN5 & 0.9239 \\
\hline KCND3 & 0.8687 \\
\hline ZNF562 & 0.8695 \\
\hline GATM & 0.9106 \\
\hline SYNPO & 0.9148 \\
\hline ZNF556 & 0.9156 \\
\hline NEGR1 & 0.8696 \\
\hline DPAGT1 & 0.9135 \\
\hline$A L G 14$ & 0.9161 \\
\hline ARNT2 & 0.9155 \\
\hline FUT9 & 0.8695 \\
\hline ZNF24 & 0.869 \\
\hline$P D P 2$ & 0.8689 \\
\hline$F A M 222 B$ & 0.9144 \\
\hline$B N C 2$ & 0.8694 \\
\hline PARP14 & 0.9151 \\
\hline$T N K S$ & 0.8688 \\
\hline STOX2 & 0.8686 \\
\hline SMARCC1 & 0.9135 \\
\hline ZNF417 & 0.9126 \\
\hline PEAK1 & 0.9162 \\
\hline$N A B P 1$ & 0.916 \\
\hline$X C R 1$ & 0.9155 \\
\hline RNF213 & 0.8689 \\
\hline PHC3 & 0.9161 \\
\hline$C B X 2$ & 0.9148 \\
\hline SWSAP1 & 0.9097 \\
\hline$C D 34$ & 0.8687 \\
\hline CYB561D1 & 0.8686 \\
\hline$M G A$ & 0.9156 \\
\hline ATP2A2 & 0.9155 \\
\hline CNTNAP2 & 0.8685 \\
\hline IGDCC 3 & 0.9216 \\
\hline MYO1H & 0.9103 \\
\hline SLCO2A1 & 0.9126 \\
\hline
\end{tabular}




\begin{tabular}{|c|c|}
\hline TMEM167A & 0.8687 \\
\hline SH3PXD2B & 0.8687 \\
\hline C4orf32 & 0.8692 \\
\hline FZD4 & 0.8685 \\
\hline PDE12 & 0.916 \\
\hline CA5A & 0.916 \\
\hline VCPIP1 & 0.8691 \\
\hline YPEL2 & 0.915 \\
\hline CADM2 & 0.869 \\
\hline SMAD2 & 0.9162 \\
\hline EIF3F & 0.9155 \\
\hline$A L G 10 B$ & 0.8695 \\
\hline RPS6KB2 & 0.9185 \\
\hline$M L X I P$ & 0.9157 \\
\hline SLC35E3 & 0.8696 \\
\hline ZDHНC21 & 0.8694 \\
\hline JAKMIP2 & 0.8689 \\
\hline SPRYD4 & 0.9162 \\
\hline RNF152 & 0.916 \\
\hline ZNF843 & 0.9137 \\
\hline MTX3 & 0.9153 \\
\hline SLC38A9 & 0.9127 \\
\hline POLE & 0.8693 \\
\hline$S C N 4 B$ & 0.9146 \\
\hline RIMKLA & 0.8691 \\
\hline RPS6KA3 & 0.9153 \\
\hline HIC1 & 0.915 \\
\hline$P A W R$ & 0.9159 \\
\hline MIEF2 & 0.9133 \\
\hline SAMD12 & 0.916 \\
\hline$I L 17 R A$ & 0.8687 \\
\hline ARL6IP6 & 0.9131 \\
\hline AMER3 & 0.9146 \\
\hline NT5DC1 & 0.8689 \\
\hline CSRNP3 & 0.8693 \\
\hline PXT1 & 0.9124 \\
\hline CLK3 & 0.8696 \\
\hline$A R I D 3 B$ & 0.9221 \\
\hline
\end{tabular}

\begin{tabular}{|c|c|}
\hline C14orf28 & 0.9215 \\
\hline ZNF154 & 0.8686 \\
\hline SOCS4 & 0.9221 \\
\hline FGD6 & 0.869 \\
\hline PLD5 & 0.9159 \\
\hline ZNF609 & 0.869 \\
\hline TSPYL5 & 0.9146 \\
\hline YOD1 & 0.9146 \\
\hline GPR157 & 0.9144 \\
\hline LRRC57 & 0.916 \\
\hline$A E N$ & 0.9132 \\
\hline NME9 & 0.9126 \\
\hline ZNF678 & 0.922 \\
\hline$R F X 7$ & 0.869 \\
\hline RNF41 & 0.8688 \\
\hline RTKN2 & 0.8685 \\
\hline MGAT4C & 0.8697 \\
\hline CREB3L2 & 0.9159 \\
\hline$R G M A$ & 0.8695 \\
\hline HHIPL1 & 0.9156 \\
\hline FIGN & 0.9237 \\
\hline PLCXD1 & 0.9155 \\
\hline MXRA7 & 0.9158 \\
\hline$P A P P A$ & 0.9223 \\
\hline C16orf72 & 0.9224 \\
\hline PLCXD3 & 0.9156 \\
\hline CEP63 & 0.9151 \\
\hline GJC1 & 0.9157 \\
\hline CALN1 & 0.8694 \\
\hline POTEC & 0.9218 \\
\hline ZNF623 & 0.8688 \\
\hline$M A C C 1$ & 0.8686 \\
\hline KREMEN1 & 0.9154 \\
\hline KCTD16 & 0.8695 \\
\hline B3GALT5 & 0.9162 \\
\hline TMPRSS2 & 0.9123 \\
\hline FAM120C & 0.8688 \\
\hline GOLGA6L4 & 0.9143 \\
\hline
\end{tabular}

\begin{tabular}{|c|c|}
\hline PCDH9 & 0.9162 \\
\hline SDR42E1 & 0.9225 \\
\hline FLRT2 & 0.9162 \\
\hline FAM43A & 0.9107 \\
\hline PURA & 0.8695 \\
\hline ZВТВ37 & 0.9237 \\
\hline TNFAIP8L1 & 0.9139 \\
\hline$R A D 51 D$ & 0.8695 \\
\hline IFNLR1 & 0.9145 \\
\hline BRCC3 & 0.9122 \\
\hline LSAMP & 0.8693 \\
\hline$L M L N$ & 0.9158 \\
\hline$P B X 1$ & 0.9158 \\
\hline C16orf52 & 0.9146 \\
\hline YTHDF3 & 0.8686 \\
\hline PIGP & 0.8693 \\
\hline IKZF1 & 0.8687 \\
\hline PTCH1 & 0.9161 \\
\hline CYP2R1 & 0.9128 \\
\hline MARC1 & 0.8688 \\
\hline ZNF555 & 0.8686 \\
\hline KPNA4 & 0.9225 \\
\hline FSD2 & 0.8686 \\
\hline PPARA & 0.9161 \\
\hline NAP1L1 & 0.9226 \\
\hline SESTD1 & 0.8691 \\
\hline TET3 & 0.9221 \\
\hline LIN28B & 0.9235 \\
\hline $\begin{array}{l}\text { TMEM256- } \\
\text { PLSCR3 }\end{array}$ & 0.9117 \\
\hline FAM122A & 0.9151 \\
\hline SHISA7 & 0.8688 \\
\hline ZC3H6 & 0.9161 \\
\hline NCR3LG1 & 0.869 \\
\hline ZNF793 & 0.8685 \\
\hline ZNF383 & 0.8689 \\
\hline CENPP & 0.8687 \\
\hline$R A L G A P A 2$ & 0.9144 \\
\hline
\end{tabular}




\begin{tabular}{|c|c|}
\hline ASAH2 & 0.9151 \\
\hline PTAR1 & 0.9224 \\
\hline$P A R V B$ & 0.8688 \\
\hline$V W C 2$ & 0.8694 \\
\hline SNTN & 0.9217 \\
\hline BEND4 & 0.916 \\
\hline$N A$ & 0.9137 \\
\hline PTPLAD2 & 0.9225 \\
\hline КСTD21 & 0.9122 \\
\hline NDUFA4 & 0.9197 \\
\hline FAM179A & 0.9159 \\
\hline PTPRT & 0.9158 \\
\hline PLEKHG4 & 0.8685 \\
\hline$R Y R 1$ & 0.8686 \\
\hline SRGAP3 & 0.9157 \\
\hline$L C O R$ & 0.8691 \\
\hline FUT4 & 0.8685 \\
\hline ZNF774 & 0.8692 \\
\hline ZNF765 & 0.9225 \\
\hline TSC22D2 & 0.869 \\
\hline ZNF605 & 0.8693 \\
\hline IPO4 & 0.9148 \\
\hline GDAP2 & 0.9224 \\
\hline TPK1 & 0.9138 \\
\hline$M A N 2 A 2$ & 0.9145 \\
\hline$H D A C 2$ & 0.869 \\
\hline SLC22A25 & 0.9138 \\
\hline WNK3 & 0.8687 \\
\hline ZKSCAN5 & 0.8685 \\
\hline TECPR2 & 0.9152 \\
\hline ZNF512B & 0.9213 \\
\hline ZNF431 & 0.9225 \\
\hline$N F 1$ & 0.8687 \\
\hline COL27A1 & 0.9144 \\
\hline POTEI & 0.9142 \\
\hline NHLRC2 & 0.9162 \\
\hline FLNA & 0.8692 \\
\hline SRGAP1 & 0.9226 \\
\hline
\end{tabular}

\begin{tabular}{|c|c|}
\hline LRRC8B & 0.8688 \\
\hline NOL $4 L$ & 0.8686 \\
\hline C6orf141 & 0.9121 \\
\hline DDI2 & 0.8689 \\
\hline TRIM33 & 0.8685 \\
\hline LRP10 & 0.9151 \\
\hline CDC42SE1 & 0.9144 \\
\hline EME2 & 0.915 \\
\hline ZNF81 & 0.8687 \\
\hline ERO1L & 0.9154 \\
\hline PLCG2 & 0.9154 \\
\hline FCHSD 1 & 0.9198 \\
\hline ZNF121 & 0.869 \\
\hline$M B P$ & 0.8694 \\
\hline MRPL42 & 0.8696 \\
\hline ZNF248 & 0.9149 \\
\hline CACNA1E & 0.8695 \\
\hline$H E L Z$ & 0.9161 \\
\hline ZKSCAN8 & 0.8691 \\
\hline$A S P H$ & 0.8691 \\
\hline ZNF26 & 0.8696 \\
\hline NRARP & 0.9106 \\
\hline ZNF587 & 0.9158 \\
\hline$M D M 4$ & 0.9162 \\
\hline IPO9 & 0.9158 \\
\hline SLC5A3 & 0.8693 \\
\hline CNOT7 & 0.8691 \\
\hline LRIG2 & 0.8695 \\
\hline МАРЗКЗ & 0.9135 \\
\hline ATG9A & 0.9146 \\
\hline EFCAB2 & 0.8689 \\
\hline CHIC1 & 0.9155 \\
\hline PHACTR4 & 0.9148 \\
\hline$P B X 2$ & 0.9108 \\
\hline$F A M 155 A$ & 0.8692 \\
\hline PSORS1C2 & 0.9099 \\
\hline$F B X 048$ & 0.8689 \\
\hline PCDHA4 & 0.9224 \\
\hline
\end{tabular}

\begin{tabular}{|c|c|}
\hline TRIM13 & 0.9158 \\
\hline SLC35B4 & 0.915 \\
\hline ZBTB10 & 0.9158 \\
\hline TMEM170B & 0.8689 \\
\hline GPR56 & 0.9148 \\
\hline C15orf59 & 0.9153 \\
\hline C5orf51 & 0.9156 \\
\hline ONECUT3 & 0.9158 \\
\hline NYNRIN & 0.9194 \\
\hline ATP10A & 0.8686 \\
\hline$P B X 2$ & 0.9108 \\
\hline PSORS1C2 & 0.9105 \\
\hline$V G L L 3$ & 0.8695 \\
\hline TRIM71 & 0.9239 \\
\hline METTL6 & 0.9151 \\
\hline$X K R 4$ & 0.9162 \\
\hline PRR22 & 0.9135 \\
\hline C17orf51 & 0.869 \\
\hline FGFR1OP & 0.8696 \\
\hline GIMAP1 & 0.9148 \\
\hline$N R A S$ & 0.915 \\
\hline$S Y N J 2 B P$ & 0.916 \\
\hline LEPROT & 0.8687 \\
\hline RPS29 & 0.8691 \\
\hline ZNF891 & 0.9226 \\
\hline VSTM5 & 0.9139 \\
\hline PEX26 & 0.9237 \\
\hline SIAH3 & 0.9158 \\
\hline$C C D C 7$ & 0.9141 \\
\hline PLXNA4 & 0.916 \\
\hline APOL6 & 0.8693 \\
\hline$P B X 2$ & 0.9108 \\
\hline$P B X 2$ & 0.9108 \\
\hline PSORS1C2 & 0.9105 \\
\hline$P B X 2$ & 0.9108 \\
\hline PSORS1C2 & 0.9105 \\
\hline KIAA0040 & 0.8685 \\
\hline$P B X 2$ & 0.9108 \\
\hline
\end{tabular}




\begin{tabular}{|ll|}
\hline ARHGEF38 & 0.9146 \\
TMEM189 & 0.8693 \\
ARHGAP8 & 0.9207 \\
AMACR & 0.913 \\
PEG10 & 0.9158 \\
NA & 0.9113 \\
MARS2 & 0.9106 \\
PRR5-ARHGAP8 & 0.9092 \\
FMN1 & 0.8694 \\
DNAH10OS & 0.8686 \\
PCDHA10 & 0.8693 \\
ATXN7L3B & 0.9224 \\
NA & 0.8685 \\
SOGA3 KIAAO408 & 0.9224 \\
NOX5 & 0.8689 \\
\hline
\end{tabular}

\begin{tabular}{|ll|}
\hline ZNF432 & 0.9142 \\
CUX1 & 0.8695 \\
P2RX5-TAX1BP3 & 0.8686 \\
ITGB3 & 0.9139 \\
$N A$ & 0.9149 \\
$R B M 15 B$ & 0.8685 \\
$X K R 7$ & 0.8688 \\
TMEM178B & 0.9225 \\
GAN & 0.924 \\
$N A$ & 0.8687 \\
$N A$ & 0.9139 \\
$C 19 o r f 84$ & 0.9112 \\
$R N F 115$ & 0.8695 \\
$Z N F 850$ & 0.9156 \\
$N A$ & 0.9201 \\
\hline
\end{tabular}

\begin{tabular}{|ll|}
\hline TRABD2B & 0.8689 \\
SLC25A53 & 0.8687 \\
NUDT3 & 0.8694 \\
GRIN2B & 0.9226 \\
ZBTB8B & 0.9225 \\
SOCS7 & 0.8689 \\
GOLGA6L9 & 0.9147 \\
ZNF280B & 0.9147 \\
DDTL & 0.9107 \\
TTYH1 & 0.9135 \\
TTYH1 & 0.9135 \\
NA & 0.9141 \\
RBFOX2 & 0.8688 \\
ZNF8 & 0.9161 \\
\hline
\end{tabular}

Table I: miRNAs implicated in the pathogenesis of glioblastoma.

\begin{tabular}{|c|c|c|c|c|c|}
\hline hsa-let-7a-1 & hsa-mir-137 & hsa-mir-181b-2 & hsa-mir-21 & hsa-mir-30c-1 & \\
\hline hsa-let-7a-2 & hsa-mir-139 & hsa-mir-181c & hsa-mir-210 & hsa-mir-30c-2 & hsa-mir-455 \\
\hline hsa-let-7a-3 & hsa-mir-142 & hsa-mir-181d & hsa-mir-218-1 & hsa-mir-31 & hsa-mir-486 \\
\hline hsa-let-7d & hsa-mir-143 & hsa-mir-183 & hsa-mir-218-2 & hsa-mir-3163 & hsa-mir-491 \\
\hline hsa-mir-101-1 & hsa-mir-145 & hsa-mir-184 & hsa-mir-22 & hsa-mir-32 & hsa-mir-504 \\
\hline hsa-mir-101-2 & hsa-mir-146a & hsa-mir-18a & hsa-mir-221 & hsa-mir-323a & hsa-mir-539 \\
\hline hsa-mir-106a & hsa-mir-146b & hsa-mir-193a & hsa-mir-222 & hsa-mir-323b & hsa-mir-7-1 \\
\hline hsa-mir-10a & hsa-mir-148a & hsa-mir-195 & hsa-mir-224 & hsa-mir-326 & hsa-mir-7-2 \\
\hline hsa-mir-10b & hsa-mir-149 & hsa-mir-196b & hsa-mir-23b & hsa-mir-328 & hsa-mir-7-3 \\
\hline hsa-mir-124-1 & hsa-mir-151a & hsa-mir-19a & hsa-mir-25 & hsa-mir-329-1 & hsa-mir-708 \\
\hline hsa-mir-124-2 & hsa-mir-153-1 & hsa-mir-19b-1 & hsa-mir-26a-1 & hsa-mir-329-2 & hsa-mir-873 \\
\hline hsa-mir-124-3 & hsa-mir-153-2 & hsa-mir-19b-2 & hsa-mir-26a-2 & hsa-mir-342 & hsa-mir-885 \\
\hline hsa-mir-125b-1 & hsa-mir-155 & hsa-mir-200 & hsa-mir-27b & hsa-mir-34a & hsa-mir-9-1 \\
\hline hsa-mir-125b-2 & hsa-mir-15a & hsa-mir-200b & hsa-mir-29a & hsa-mir-367 & hsa-mir-9-2 \\
\hline hsa-mir-1260a & hsa-mir-16-1 & hsa-mir-205 & hsa-mir-29c & hsa-mir-376a-1 & hsa-mir-9-3 \\
\hline hsa-mir-128-1 & hsa-mir-16-2 & hsa-mir-206 & hsa-mir-302a & hsa-mir-376a-2 & hsa-mir-92a-1 \\
\hline hsa-mir-128-2 & hsa-mir-17 & hsa-mir-208a & hsa-mir-302b & hsa-mir-381 & hsa-mir-92a-2 \\
\hline hsa-mir-1305 & hsa-mir-181a-1 & hsa-mir-208b & hsa-mir-302c & hsa-mir-425 & hsa-mir-95 \\
\hline hsa-mir-130a & hsa-mir-181a-2 & hsa-mir-20a & hsa-mir-302d & hsa-mir-451a & hsa-mir-99a \\
\hline hsa-mir-134 & hsa-mir-181b-1 & hsa-mir-20b & hsa-mir-30a & hsa-mir-452 & \\
\hline
\end{tabular}


The genes with remarkable expression profile differences between glioblastoma and normal brain tissues among glioblastoma-associated ceRNAs involving T-UCR were defined. Expression of PBX3 gene was significantly higher and NRXN3 gene expression was remarkably lower in glioblastoma than in normal brain tissues according to the current analysis. On the other hand, the other genes did not show any remarkable expression differences (Table III).

Table II: The glioblastoma-associated ceRNAs that match the genes containing T-UCR in the exonic regions.

\begin{tabular}{|ccc|}
\hline uc.378 & 251 & NRXN3 \\
uc.184 & 230 & CPEB4 \\
uc.33 & 312 & PTBP2 \\
uc.414 & 246 & THRA \\
uc.280 & 220 & PBX3 \\
uc.393 & & CLK3 \\
\hline
\end{tabular}

Table III: Expression values of ceRNAs with T-UCR that are associated with glioblastoma in normal brain tissues and glioblastoma.

\begin{tabular}{|lll|}
\hline NRXN3* & $\mathbf{1 . 8 4}$ & \\
CPEB4 & 14.04 & $\mathbf{1 8 . 4 1}$ \\
PTBP2 & 12.1 & 11.54 \\
THRA & 99.62 & 11.96 \\
PBX3* & $\mathbf{1 9 . 5 4}$ & 144.88 \\
CLK3 & 32.92 & $\mathbf{3 . 2 9}$ \\
& & 29.86 \\
\hline
\end{tabular}

* shows remarkably differential expression profile between normal brain tissues and glioblastoma
The statistical analysis of the relationship between PBX3 and NRXN3 genes and glioblastoma multiforme was carried out via GEPIA database. It was determined that PBX3 and NRXN3 genes were significantly correlated with glioblastoma based on the Spearman correlation analysis (Figure 1) $(\mathrm{p}=0.0014$; $\mathrm{R}=-$ $0.17)$.

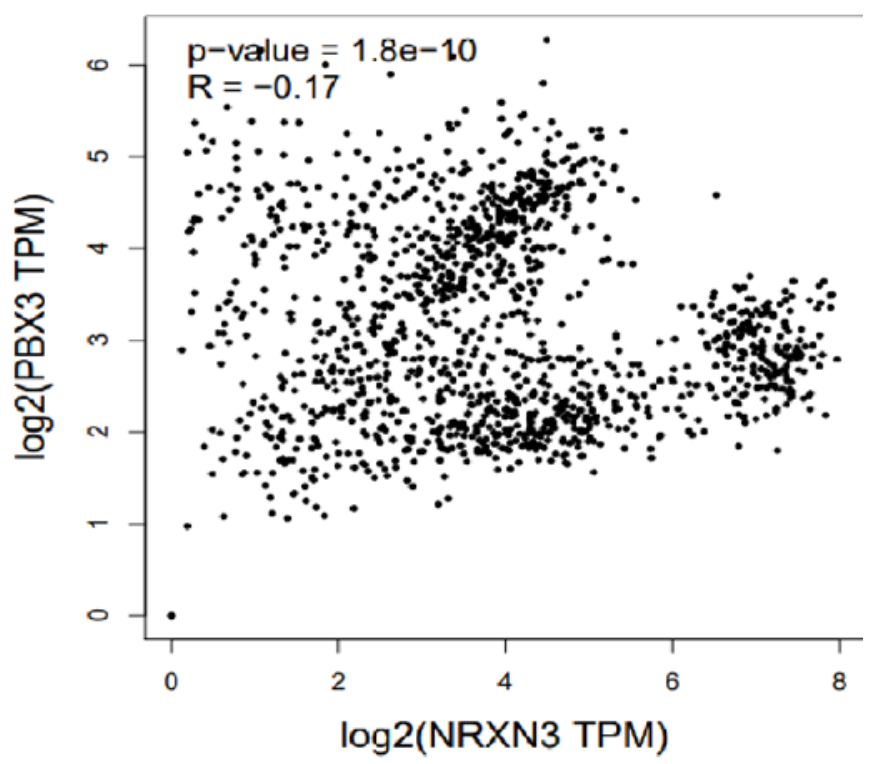

Figure 1: The relationship of NRXN3 and PBX3 genes with glioblastoma.

\section{DISCUSSION}

Glioblastoma which is the most frequent and aggressive form of primary malignancies in adult human brains is characterized by tumor heterogeneity, diffuse invasion, drug resistance, and rapid growth. It has been clarified that miRNAs are implicated in tumorigenesis. Moreover, it has been observed that expression levels of miRNAs are differed between pathological and normal tissues. Recent studies have subclassified glioblastoma into five clinically and genetically distinct subtypes according to miRNA expression profiles and it has been supposed that miRNAs are important for the phenotypic characteristics of the subclasses ${ }^{16,17}$. The median survival time of patients with GBM is approximately 14 to 16 months despite standard treatment options and there is no cure at present. In recent years, 
studies in this field have been focused on the identification of new targets for diagnostics and therapeutics for GBM. It is supposed that detection and quantifying miRNAs in serum and tissues will become a standard tool for diagnosis and prognosis of GBM and have a great potential for personalized treatment strategies ${ }^{18}$. In this regard, based on the idea that miRNAs are implicated in the pathogenesis of glioblastoma, we aimed to determine novel molecular biomarkers for GBM through in silico analysis that uses glioblastoma-specific microRNAs, identifies their combinatorial target genes which have potential ceRNA activities. In this study, 118 microRNAs correlated with glioblastoma were obtained from miRTarBase database (Table I). The genes with ComiR score greater than 0.8685 were listed through 1016 genes that are simultaneously targeted by these 118 miRNAs. The genes with T-UCR in their exonic regions were selected based on the study of Bejerano et al.14. Subsequently, the genes which show potential ceRNA activities were extracted (Table II). Then, the genes with remarkable expression diffrences between GBM and normal brain tissues were extracted from glioblastomaassociated ceRNAs that include T-UCR. While PBX3 gene was highly expressed in GBM than in normal brain tissues, NRXN3 gene was significantly less expressed in GBM than in normal brain tissues according to the analysis in this study. On the other hand, other genes did not show any significant differences in expression pattern. According to the findings of the Spearman correlation analysis, PBX3 and NRXN3 genes were shown to have remarkable relationship with GBM.

PBX3 is a member of Pre-B-cell leukemia homeobox family and implicated in early development and several biological processes in adulthood. The location of PBX3 gene is on chromosome 9q33.3. PBX3 as a transcription factor shows a stable interaction with DNA and binds to DNA with a consensus sequence (TGATTGATTTGAT). It has been demonstrated that PBX3 is commonly associated with cancer and overexpressed in several types of cancers such as hematological malignancies and colorectal cancers. Moreover, PBX3 activates numerous signaling pathways such as MAPK/ERK signaling pathway. PBX3 functions as an oncogene and is implicated in the regulation of biological functions such as stimulating proliferation, colony formation, cell survival, and invasion ${ }^{19,20}$. It has been demonstrated that PBX3 is upregulated in gastric cancer cells and apoptosis is induced by targeting PBX3 gene in gastric cancer ${ }^{20}$. In a study conducted with glioma cell lines, it has been shown that PBX3 was overexpressed ${ }^{21}$. Xu et al. reported that $\mathrm{PBX} 3$ was significantly associated with invasion of GBM cells and mesenchymal transition ${ }^{22}$.

Neurexins (NRXNs) are a family of neuronalspecific cell surface proteins and they are implicated in cell recognition and adhesion. Moreover, the presynaptic terminal proteins are involved in synaptogenesis, neurotransmitter release and synaptic transmission and are also essential for the development and function of synapses. NRXN genes are differentially spliced into numerous isoforms ${ }^{23,24}$. It is known that FoxQ1 as a potential oncogene may induce tumor cell proliferation and migration by targeting NRXN3 gene in a direct way ${ }^{25}$. It has been reported that FoxQ1 stimulated cell proliferation and migration of glioma by suppressing NRXN3 gene and suggested that NRXN3 gene might be a tumor suppressor ${ }^{24}$. In the study conducted with breast cancer patients, $G$ allele carriers in rs10146997 of NRXN3 gene was statistically related to the development of breast cancer ${ }^{26}$. It has been reported that NRXN3 gene expression was downregulated in the samples of GBM 27 .

NRXN3 and PBX3 genes were associated with GBM in this present study and they were 
suggested to have potential roles in carcinogenesis. It has been supposed that NRXN3 acts as a tumor suppressor gene and its expression is decreased in GBM according to the analysis in this study. On the other hand, PBX3 gene is suggested to function as an oncogene and is upregulated in GBM according to the in silico analysis.

\section{CONCLUSION}

The present study investigated the correlation of NRXN3 and PBX3 genes with GBM and this study supports the potential roles for the genes in the pathogenesis of glioblastoma. Additionally, further in vivo and in vitro studies are needed in order to elucidate tumor suppressor role of NRXN3 and oncogenic activity of PBX3 in GBM.

Ethics Committee Approval: This study did not require any ethical approval.

Declaration of Conflicting Interests: The authors declare that they have no conflict of interest.

Financial Disclosure: No financial support was received.

\section{REFERENCES}

1. Yool AJ, Ramesh S. Molecular targets for combined therapeutic strategies to limit glioblastoma cell migration and invasion. Front Pharmacol. 2020; 11: 358.

2. Haar CP, Hebbar P, Wallace GC, et al. Drug resistance in glioblastoma: A mini review. Neurochem Res. 2012; 37: 1192-200.

3. Yılmaz A, Altug F, Duz E, et al. Treatment options and effects of survival in glioblastoma multiforme. J Kartal TR. 2012; 23: 25-9.

4. Laffont B, Rayner KJ. MicroRNAs in the pathobiology of atherosclerosis. CJC. 2017; 33: 31324.

invasion program in glioblastoma. J Exp Clin Cancer Res. 2019; 38.
5. Witwer KW, Halushka MK. Toward the promise of microRNAs- Enhancing reproducibility and rigor in microRNA research. RNA Biol. 2016; 13: 1103-16.

6. Sherin K, Nair AS. Review of computational prediction of competing endogenous RNA. J Proteom Bioinform. 2019; 12.

7. Qi M, Yu B, Yu H, et al. Integrated analysis of a ceRNA network reveals potential prognostic IncRNAs in gastric cancer. Cancer Med. 2020; 9: 1798-817.

8. Fassan $M$, Dall'Olmo $L$, Galasso $M$, et al. Transcribed ultraconserved noncoding RNAs (TUCRs) are involved in Barret's esophagus carcinogenesis. Oncotarget. 2014; 5: 7162-71.

9. Huang HY, Lin YCD, Li J, et al. miRTarBase 2020: updates to the experimentally validated microRNAtarget interaction database. Nucleic Acids Res. 2020; 48: D148-D154.

10. Ergun S. In silico analysis of biomarker potentials of miRNA-mediated ceRNAs in prostate cancer. Dicle Med J. 2018; 45: 415-29.

11. Coronnello C, Benos PV. ComiR: combinatorial microRNA target prediction tool. Nucleic Acids Res. 2013; 41: W159-W164.

12. Us Altay D, Ergun S. In silico analysis of biomarker potentials of miRNA mediated ceRNAs in gastric neoplasms. MBSJHS. 2019; 5: 106-19.

13. Avsar 0. Analysis of miRNA-mediated ceRNAs in the pathogenesis of renal cell carcinoma: An in silico approach. HJSE. 2020; 7: 223-38.

14. Bejerano G, Pheasant M, Makunin I, et al. Ultraconserved elements in the human genome. Science. 2004; 304:1321-5.

15. Tang Z, Li C, Kang B, et al. GEPIA: a web server for cancer and normal gene expression profiling and interactive analysis. Nucleic Acids Res. 2017; 45: W98-W102.

16. Zhao Y, Huang W, Kim TM, et al. MicroRNA-29a activates a multi-component growth and

17. Touat M, Idbaih A, Sanson M, et al. Glioblastoma targeted therapy: updated approaches from recent biological insights. Ann Oncol. 2017; 28: 1457-72. 
18. Shea A, Harish V, Afzal Z, et al. MicroRNAs in glioblastoma multiforme pathogenesis and therapeutics. Cancer Med. 2016; 5: 1917-46.

19. Morgan R, Pandha HS. PBX3 in cancer. Cancers. $2020 ; 12$.

20. Li YS, Zou Y, Dai DQ. MicroRNA-320a suppresses tumor progression by targeting $\mathrm{PBX} 3$ in gastric cancer and is downregulated by DNA methylation. World J Gastrointest Oncol. 2019; 11: 842-56.

21. Pan C, Gao H, Zheng N, et al. Mir-320 inhibits the growth of glioma cells through downregulating PBX3. Biol Res. 2017; 50.

22. $\mathrm{Xu} \mathrm{X}, \mathrm{Bao} \mathrm{Z}$, Liu $\mathrm{Y}$, et al. PBX3/MEK/ERK1/2/LIN28/let-7b positive feedback loop enhances mesenchymal phenotype to promote glioblastoma migration and invasion. J Exp Clin Cancer Res. 2018; 37: 158.

23. Harkin LF, Lindsay SJ, Xu Y, et al. Neurexins 1-3 each have a distinct pattern of expression in the early developing human cerebral cortex. Cereb Cortex. 2017; 27: 216-32.

24. Sun HT, Cheng SX, Tu Y, et al. FoxQ1 promotes glioma cells proliferation and migration by regulating NRXN3 expression. PLoS One. 2013; 8: e55693.

25. Xiang XJ, Deng J, Liu YW, et al. Mir-1271 inhibits cell proliferation, invasion and EMT in gastric cancer by targeting FOXQ1. Cell Physi

ol Biochem. 2015; 36: 1382-94.

26. Kusinska R, Gorniak P, Pastorczak A, et al. Influence of genomic variation in FTO at 16q12.2, MC4R at 18q22 and NRXN3 at 14q31 genes on breast cancer risk. Mol Biol Rep. 2012; 39: 2915-19.

27. Yang $Q$, Wang $R$, Wei $B$, et al. Gene and microRNA signatures are associated with the development and survival of glioblastoma patients. DNA Cell Biol. 2019; 38: 688-99. 